\title{
Multi-detector Computed Tomography versus Digital Panoramic Radiography for Preoperative Evaluation of Jaw Bones before Dental Implantation
}

Tarek Mohamed M. Mansour ${ }^{1 *}$, Mohamad Hasan Tawfik ${ }^{2}$, Mohamed M. El-Barody ${ }^{3}$, Ibrahim M. Mwafey ${ }^{4}$, Ahmed Okasha ${ }^{5}$

${ }^{1}$ Department of Radio-diagnosis, ${ }^{4}$ Department of Oral Medicine and Periodontology Diagnosis and Oral Radiology, Faculty of Medicine, Al-Azhar University, Assuit, ${ }^{2}$ Department of Radio-diagnosis, Faculty of Medicine, Al-Azhar University, Cairo, ${ }^{3}$ Department of Radio-diagnosis, South Egypt Cancer Institute, Assuit University, Assuit, ${ }^{5}$ Department of Radio-Diagnosis, Faculty of Medicine, South Valley University, Qena, Egypt

*Correspondence author: Tarek Mohamed M. Mansour, E-mail: drtarekrad@gmail.com, Mobile: (+20)01006237731

\begin{abstract}
Background: To evaluate and compare preoperatively multi-detector computed tomography and digital panoramic radiography of jaw bones in patients submitted to dental implant restoration.

Patients and methods: We conducted a diagnostic test accuracy study, Al-Azhar University Hospital Assuit. Thirty patients, with partial edentulous areas, were enrolled in the study and they were 11 females and 19 males with mean age of 37 years. Patients were included if they were systemically healthy, at least 18 years old, missing a single or multiple teeth, and their crestal residual ridge width ranging from 3 to $5 \mathrm{~mm}$.

Results: A higher Cronbach's alpha for the multi-detector computed tomography (MDCT) modality, as well as a lower reliability of error were found. Moreover, unlike digital panorama radiography, MDCT obtained length and width could predict the implant length and diameter. These results highlight the superiority of MDCT to digital panorama radiography in the preoperative assessment before dental implantation.

Conclusion: Superiority of MDCT to digital panorama radiography in determining the implant site parameters before dental implantation. Future studies should consider reducing the cost of MDCT and improving its accessibility in low-resource settings.
\end{abstract}

Keywords: MDCT, Radiography, Panorama.

\section{INTRODUCTION}

Partial or total edentulism is a major health problem that affects health related quality of life like self-esteem, dietary intake, speech and alters food taste, and preference. Poor dietary intake will increase the risk of cardiovascular disease and cancer (1-3). The WHO declared that total or partial absence of natural teeth is a public health concern and recent data indicated possible increases in prevalence in the next decades ${ }^{(4)}$. In addition, loss of teeth is frequent in $30 \%$ of adults and the percentage increased in low socioeconomic class ${ }^{(1-3)}$. In Egypt, edentulism affects a large proportion of the population ${ }^{(5,6)}$.

Osseointegrated implants are an alternative method to healthy and comfortable denture that acts by mechanical propagation and helps in charges distribution ${ }^{(7,8)}$. Radiographic methods play a major role in assessing the bony support for endosseous dental implants. However, they are often inaccurate and have disadvantages like bad visualization for anatomic structures, superimposition, and distortion (9,10). Volumetric techniques (as Multi-detector computed tomography (MDCT) and cone beam computed tomography (CBCT)) are more accurate than conventional radiography, especially if the field is less than $15 \mathrm{~mm}$ high ${ }^{(11)}$. These techniques are effective in assessing the bone quantity in three dimensions, bone quality and determine the location of significant adjacent structures like dental inferior nerve, mental foramen, mandibular canal, maxillary sinus and incisive foramen ${ }^{(11-13)}$.

The present study was designed to evaluate and compare preoperatively multi-detector computed tomography and digital panoramic radiography of jaw bones in patients submitted to dental implant restoration.

\section{PATIENTS AND METHODS}

1. Study design and patients selection: We conducted a diagnostic test accuracy study, Al-Azhar University Hospital Assuit. Thirty patients, with partial edentulous areas, were enrolled in the study and they were 11 females and 19 males with mean age of 37 years. Patients were included if they were systemically healthy, at least 18 years old, missing a single or multiple teeth, and their crestal residual ridge width ranging from 3 to $5 \mathrm{~mm}$. Patients were excluded from the study if they had any of the following: alcoholic, smoking, drug abused, currently or from three months ago on certain medications like bisphosphonates or steroids, pregnant or receiving contraceptive pills, had obvious undercut on the labial cortical plate or perforated 
and/or lost labial bony plate, cases of complicated wound healing like uncontrolled diabetes mellitus or cases w4ith lowered possibility of bone regeneration like osteoporosis or Paget's disease, uncontrolled periodontal cases or oral diseases, and excluded if had history of grafting procedures at area of interest or history of radiotherapy in head and neck region.

2. Ethical approval: The study protocol was approved by the Medical Research Ethics Committee at the Institutional Review Board of Al-Azhar University. The study was conducted at the Outpatient Clinic, Oral Medicine and Periodontology Department. All enrolled cases gave an informed consent after understanding the aim of the study and before any study procedure.

3. Study procedure: In one visit each patient was exposed to two radiographic machines for one time only: 1. Sirona DP radiography and 2. MDCT gives (para-axial) reformatted view to generate MD computed tomography (reformatted para-axial cuts), and digital X-ray radiographic panorama. The following parameters were measured for each patient: alveolar margin length and width in panorama and MDCT para-axial cuts, and post-surgical length and diameter of the implant. All measurements were conducted by two blinded observers to obtain an accurate data.

Through MDCT imaging of the jaws and by using the same protocol that routinely used in clinical practice, the skulls were imaged in a spiral 16-row MDCT scanner (Light Speed 2014, Siemens, US). The jaws axial sectional images with $80 \mathrm{~mA}$ and 120 $\mathrm{kV}$ were obtained with the scanning plane for the mandible parallel to the long axis of the body of the mandible and the scanning plane for the maxilla parallel to the hard palate. The acquisition time for the axial slices was 1 rotation (16 slices) per second. The thickness of slice was $1.25 \mathrm{~mm}$ with $1.25 \mathrm{~mm}$ spacing and the beam pitch was 0.562:1. In addition, the display FOV (DFOV) was $16.7 \mathrm{~cm}$, with a matrix size of 512 (calculated pixel size: $0.33 \mathrm{~mm}$ ). We used the reformatting software (DentaScan Plus, Siemens, US) to gain the transverse para-axial images of the jaws $2 \mathrm{~mm}$ in thickness (the standard thickness obtained from the software program).

All MDCT scans were checked to ensure consistent head placement (the occlusal plane parallel to the floor). In addition, the cross sectional views perpendicular to the alveolar ridge were taken in the middle of maxillary right central incisor, lateral incisor, and canine regions. Respectively absence or presence of buccal undercut was assessed in a right maxillary lateral incisor and canine.

\section{Statistical analysis}

Results were analyzed using IBM SPSS (Statistical Package for the Social Sciences) software (version 20 for windows). A difference was considered significant when the $p$ value was $<0.05$. The Kolmogorov-Smirnov test was used to verify the normality of distribution. Quantitative data were described using range (minimum and maximum), mean \pm standard deviation, while categorical variables were reported as frequency (\%). The following tests were used when appropriate: one way ANOVA with post-hoc LSD test, multiple regression analysis, and Cronbach's alpha $(\alpha)$.

\section{RESULTS}

Baseline characteristics: This study included thirty systemically healthy patients (11 females and 19 males) who ranged in age between 23 and 50 years (mean age: 37 years) (Table 1).

Table (1): Distribution of the gender and age between the study groups.

\begin{tabular}{|c|l|c|}
\hline \multicolumn{2}{|c|}{ Characteristics } & N (\%) \\
\hline Age (years) & 20 YS & $1(3.3)$ \\
\cline { 2 - 3 } & 58 YS & $1(3.3)$ \\
\cline { 2 - 3 } & $21-30$ YS & $3(10)$ \\
\cline { 2 - 3 } & $31-40$ YS & $13(43.3)$ \\
\cline { 2 - 3 } & $41-50$ YS & $9(30)$ \\
\cline { 2 - 3 } & $51-57$ YS & $3(10)$ \\
\hline Gender & Male & $19(63.3)$ \\
\cline { 2 - 3 } & Females & $11(36.7)$ \\
\hline
\end{tabular}

Data are frequency (Percentage).

\section{Comparison between Observers:}

There was insignificant difference between the 2 observers (O1 and $\mathrm{O} 2)$ regarding the anthropometric measurements of MD length and width and DP length and width (Table 2). 
Table (2): Means and standard deviations of the anthropometric measurements of MD length and width and DP Length and width by observers 1 and 2.

\begin{tabular}{|c|c|c|c|c|}
\hline & $\begin{array}{c}\text { Observer 1 } \\
(\mathbf{N}=\mathbf{3 0})\end{array}$ & $\begin{array}{c}\text { Observer 2 } \\
(\mathbf{N}=\mathbf{3 0})\end{array}$ & $\boldsymbol{F}$ & $\boldsymbol{P}$ \\
\hline D.P Length & $10.50 \pm 2.25$ & $10.25 \pm 2.05$ & 0.169 & 0.68 \\
\hline D.P Width & $4.217 \pm 0.7$ & $4.13 \pm 0.72$ & 0.217 & 0.64 \\
\hline M.D Length & $14.72 \pm 2.92$ & $14.79 \pm 3.07$ & 0.007 & 0.93 \\
\hline M.D Width & $6.72 \pm 1.61$ & $6.74 \pm 1.52$ & 0.003 & 0.95 \\
\hline
\end{tabular}

\section{Comparison between MD and PD measurements:}

We observed marked increases in mean implant site length and mean implant site width traced from observer $1 \mathrm{MD}$ view and observer $1 \mathrm{DP}$ view and the difference was a statistically significant difference. Table 3 shows that there were marked increases in mean implant site length and mean implant site width traced from observer 2 MD view and observer 2 DP view and the difference was a statistically significant difference.

Table (3): Means and standard deviations of the anthropometric measurements of the MD and DP by observers 1 and 2 (O1 and $\mathrm{O} 2)$.

\begin{tabular}{|c|c|c|c|}
\hline M.D length O1 & D.P length O1 & F & $\boldsymbol{P}$ \\
\hline $\mathbf{1 4 . 7 2 7} \pm \mathbf{2 . 9 2}$ & $10.503 \pm 2.25$ & 39.3 & $<0.01$ \\
\hline M.D width O1 & D.P width O1 & F & $\boldsymbol{P}$ \\
\hline 6.723 $\pm \mathbf{1 . 6 1}$ & $4.217 \pm 0.7$ & 60.59 & $<0.01$ \\
\hline M.D length O2 & D.P length O2 & F & $\boldsymbol{P}$ \\
\hline M.79 $\pm \mathbf{3 . 0 7}$ & $10.250 \pm 2.051$ & 39.24 & $<0.01$ \\
\hline 6.74 $\pm \mathbf{1 . 5 2}$ & D.P width O2 & F & $\boldsymbol{P}$ \\
\hline
\end{tabular}

In addition, the split plot ANOVA showed no significant difference between O1 length subgroup (DP and MD) and the $\mathrm{O} 2$ subgroup (DP and MD) as the $\mathrm{F}=1.7(p=0.18)$. There was a little marginal effect between $\mathrm{O} 1$ and $\mathrm{O} 2$, as well as mild reduced DP lengths from $\mathrm{O} 1$ to $\mathrm{O} 2$ while the MD length from $\mathrm{O} 1$ to $\mathrm{O} 2$ was quite constant (Fig. 1). Moreover, no interaction between $\mathrm{O} 1$ and $\mathrm{O} 2$ was observed.

A

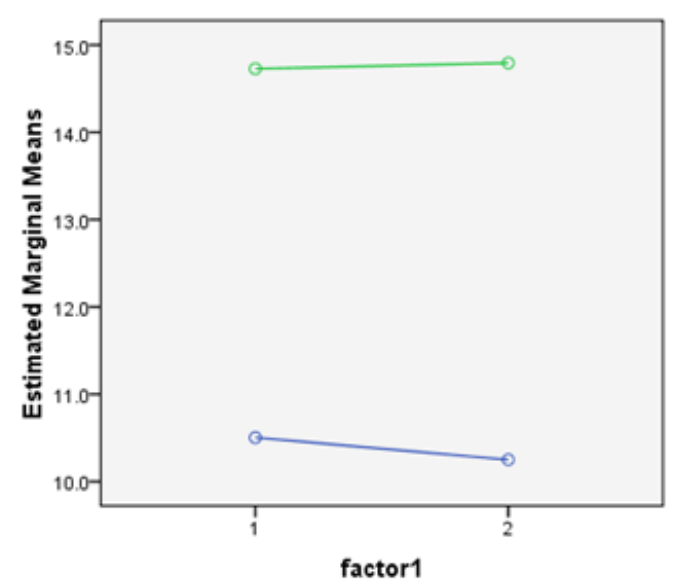

B

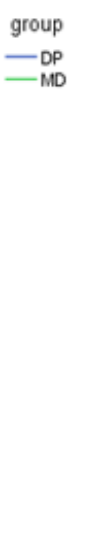

Estimated Marginal Means of MEASURE_1

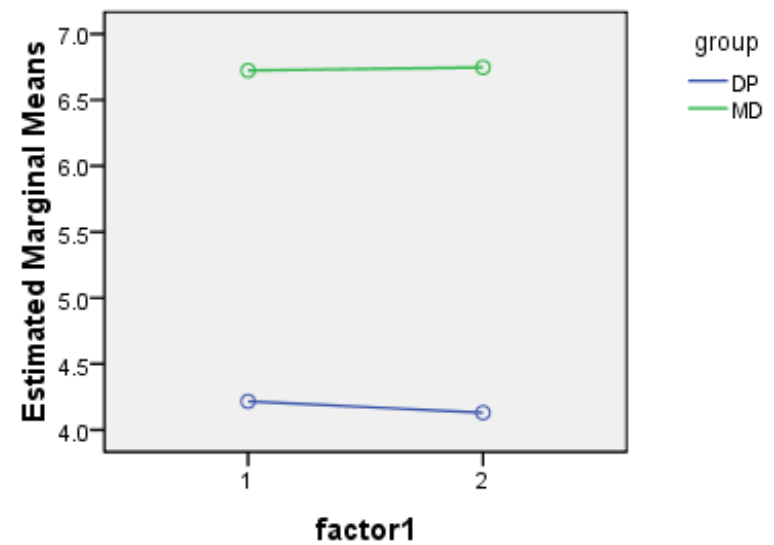

Fig. (1 A and B): shows little marginal effects between observer 1 and observer 2.

The split ANOVA test showed no statistically difference between O1 width subgroup (DP and MD) and the O2 width subgroup (DP and MD) as $\mathrm{F}=1.28(p=0.26)$. There was a little marginal effect between the $\mathrm{O} 1$ and the $\mathrm{O} 2$ as regard the implant site width as well as mild reduced DP width from $\mathrm{O} 1$ to $\mathrm{O} 2$ while the MD width from $\mathrm{O} 1$ to $\mathrm{O} 2$ was quite constant. Moreover, no interaction between $\mathrm{O} 1$ and $\mathrm{O} 2$ was observed.

\section{Comparison between real implant parameters and radiographic parameters:}

There were significant differences between implant length and average MD length (O1 and $\mathrm{O} 2)$, and implant diameter and average MD width (O1 and O2) (Table 4). 
Table (4): Means and standard deviations of the anthropometric measurements of the implant length and diameter and average DP/MD length.

\begin{tabular}{|c|c|c|c|}
\hline Implant length & Average DP length & $\boldsymbol{F}$ & $\boldsymbol{P}$ \\
\hline $14.31 \pm 2.19$ & $10.37 \pm 2.34$ & 1.07 & 0.478 \\
\hline Implant diameter & Average DP width & $\mathbf{F}$ & $\boldsymbol{P}$ \\
\hline $6.38 \pm 1.26$ & $4.17 \pm 0.69$ & 2.06 & 0.120 \\
\hline Implant length & Average MD length & $\mathbf{F}$ & $\boldsymbol{P}$ \\
\hline $14.31 \pm 2.19$ & $14.76 \pm 2.96$ & 17.10 & 0.02 \\
\hline Implant diameter & Average MD width & $\mathbf{F}$ & $\boldsymbol{P}$ \\
\hline $6.38 \pm 1.26$ & $6.73 \pm 1.55$ & 13.43 & 0.03 \\
\hline
\end{tabular}

A Cronbach's alpha of 0.897 showed that all variables can measure the same construct. In addition, the inter-item correlation matrix was more in the MD group. However, the reliability of error was better (0.858 and 0.088$)$ for MD length and width, respectively. However, it was worse in the DP group ( 0.875 and 0.898 for length and width, respectively), (Fig. 2).
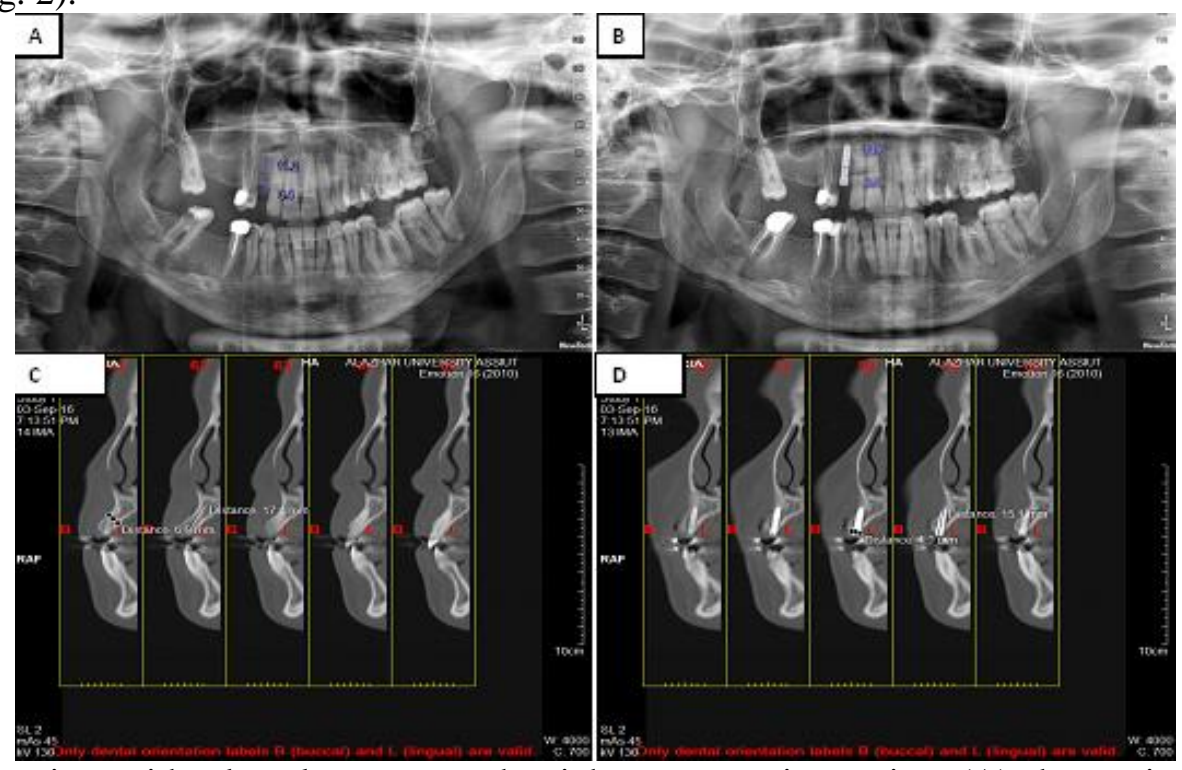

Fig. (2): Female patient with edentulous area at the right upper canine region, (A) shows single extracted right upper canine selected for preoperative evaluation of dental implant placement with digital panoramic measurements of length, width of right upper alveolar bone, (B) showing digital panoramic radiography after implant placement at the right upper canine edentulous area with measurement of length, diameter of implant, (C) showing MDCT cross sectional images with measurements of length and width of the alveolar bone of selected patient for preoperative evaluation of dental implant placement, (D) showing MDCT cross sectional images with measurements of length and width of the implant.

Prediction value for MD CT view: Table 5 shows that DP length could not predict the implant length (target parameter), while the average MD length could predict the implant length. The average MD length and width could predict the implant length and diameter. These results highlight the value of MDCT dental examination before implant surgery. Fig. 3 shows that strong linear correlations between the implant length plotted in the $\mathrm{x}$ axis and the average M.D length plotted in the Y axis (Fig. 4).

Table (5): By using meta-regression analysis; using the average D.P length that can predict the implant length as target parameter.

\begin{tabular}{|l|c|}
\hline \multicolumn{1}{|c|}{ Variables } & $\boldsymbol{P}$ - value \\
\hline D.P length. $^{(\mathbf{0 1}, \mathbf{O 2})}$ & 0.842 and 0.609 \\
\hline M.D length. $^{(\mathbf{0 1}, \mathbf{0 2})}$ & 0.023 and 0.05 \\
\hline M.D width. $^{(\mathbf{0 1}, \mathbf{0 2})}$ & 0.05 and 0.03 \\
\hline D.P width. $^{(\mathbf{0 1}, \mathbf{0 2})}$ & 0.391 and 0.716 \\
\hline
\end{tabular}


implant length
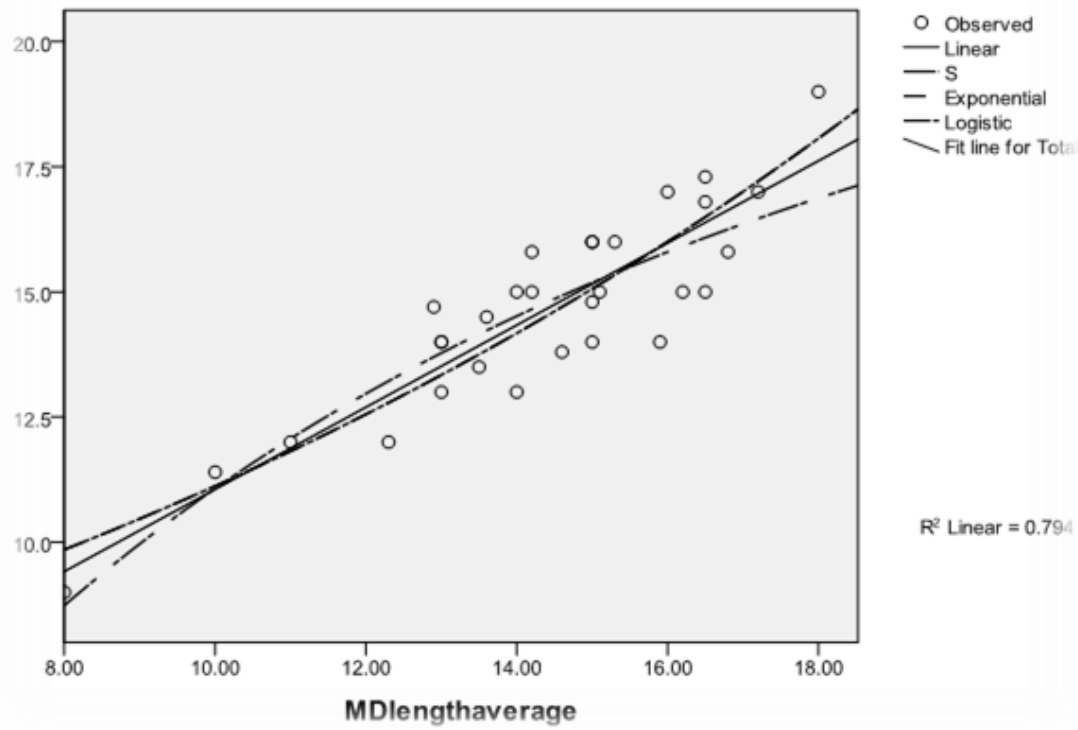

$R^{2}$ Linear $=0.79$

Fig. (3): Shows strong linear correlations between the implant length plotted in the $\mathrm{x}$ axis and the average M.D length plotted in the $\mathrm{Y}$ axis $(\mathrm{r}=0.79$ and $p<0.01)$.
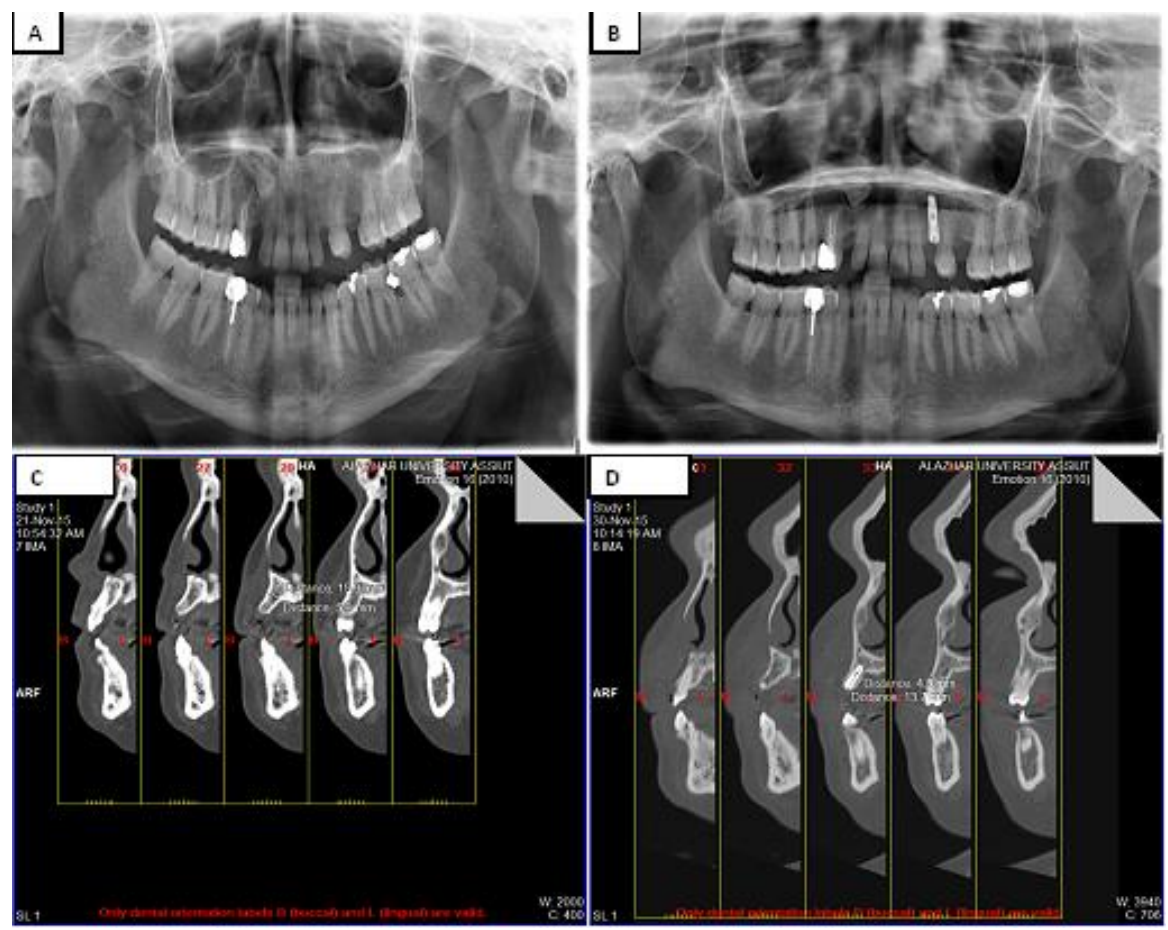

Fig. (4): Female patient 32 years old with edentulous area at the left upper canine tooth. (A) shows single extracted left upper canine selected for preoperative evaluation of dental implant placement, (B) showing digital panoramic radiography after implant placement at the left upper canine edentulous area, (C) showing MDCT cross sectional images with measurements of length and width of the alveolar bone of selected patient for preoperative evaluation of dental implant placement, (D) showing MDCT cross sectional images with measurements of length and width of the implant. 


\section{DISCUSSION}

The current study aimed to compare the diagnostic value of MDCT versus digital panoramic radiography in the preoperative assessment before dental implantation. Our data showed a higher Cronbach's alpha for the MD modality, as well as a lower reliability of error. Moreover, unlike DP radiography, MD obtained length and width could predict the implant length and diameter. These results highlight the superiority of MDCT to DP radiography in the preoperative assessment before dental implantation.

Few data exist in the literature about the comparison between DP and MDCT imaging. Previous studies revealed that high-resolution $\mathrm{CT}$ can produce crosssectional, panoramic and 3D reformatted images of the alveolar bone, generating accurate data on the bone height and width of the alveolar ridge ${ }^{(\mathbf{1 4}, 15)}$. Other advantages include elimination of superimpositions, contrast resolution, and the ability to further image projections or planar reformations (11-13).

However, to be fair, former studies reported some limitations for the MDCT modality. These include high radiation dose, reduced image quality by metallic artifacts, the high cost and limited accessibility to the procedure ${ }^{(\mathbf{1 6 - 1 8})}$. Therefore, other modalities were developed to overcome such limitations. The cone beam computed tomography (CBCT) is a newer modality that showed higher accuracy in the assessment of implant site dimensions in several prior studies; however, its images have increased noise and scatter compared with MDCT images, which lowers their contrast resolution ${ }^{(19-21)}$. Future studies should include CBCT in the comparison to determine the optimal modality for this assessment.

In comparison to previous studies, an investigation by Lam and colleagues showed that 2D CT had higher accuracy in measuring implant site parameters, especially when the bone height measurements were less than $15 \mathrm{~mm}{ }^{(11)}$. Another study by Pawelzik et al. ${ }^{(22)}$, revealed that MDCT exhibited higher accuracy than DP radiography in the preoperative assessment of impacted mandibular third molars. Saavedra-Abril and colleagues published a comprehensive review on the full technique and applications of multiscan CT in dental implant practice ${ }^{(23)}$.

We acknowledge some limitations to the current study. Frist, the small sample size limits the generalizability of our findings. Second, the study focused on the accuracy in measuring implant site parameters, while we did not focus on image resolution and assessment of artifacts. Third, we also did not correlate our findings to postoperative outcomes, which could be useful information for practicing dentists. Future studies should use larger sample size and pay attention to the aforementioned outcomes.

\section{CONCLUSION}

In conclusion, the current study showed superiority of MDCT to DP radiography in determining the implant site parameters before dental implantation. Future studies should consider reducing the cost of MDCT and improving its accessibility in lowresource settings.

\section{REFERENCES}

1. Tyrovolas S, Koyanagi A, Panagiotakos D et al. (2016): Population prevalence of edentulism and its association with depression and self-rated health. Scientific reports, 6: 37083-86.

2. Cooper L (2018): Edentulism and the demise of dentures: Facts and fallacies, in mandibular implant prostheses. Springer. Pp. 3-10.

3. Roberto L, Crespo T, Monteiro-Junior $\mathbf{R}$ et al. (2019): Sociodemographic determinants of edentulism in the elderly population: A systematic review and meta- analysis. Gerodontology, 36(4):325-337.

4. Preet K, Williams J (2016): Common risk factors and edentulism in adults, aged 50 years and over, in China, Ghana, India and South Africa: results from the WHO Study on global ageing and adult health (SAGE). BMC Oral Health, 17(1): 29-29.

5. Polzer I, Schimmel M, Müller F et al. (2010): Edentulism as part of the general health problems of elderly adults. International Dental Journal, 60(3): 143-155.

6. Petersen F, Buckner C, Appelbaum F et al. (1992): Etoposide, cyclophosphamide and fractionated total body irradiation as a preparative regimen for marrow transplantation in patients with advanced hematological malignancies: a phase I study. Bone Marrow Transplantation, 10(1): 83-88.

7. van Steenberghe $D$, Lekholm $U$, Bolender $C$ et al. (1990): The applicability of osseointegrated oral implants in the rehabilitation of partial edentulism: A prospective multicenter study on 558 fixtures. International Journal of Oral \& Maxillofacial Implants, 5(3): 272-281.

8. Lindh, T, Gunne J, Tillberg A et al. (1998): A meta- analysis of implants in partial edentulism. Clinical Oral Implants Research, 9(2): 80-90.

9. Okşayan R, Asarkaya B, Palta $N$ et al. (2017): Effects of edentulism on mandibular morphology: evaluation of panoramic radiographs. The Scientific World Journal, 2014: 1-5.

10. Smith C, Koumjian J (2018): Classification system for complete edentulism. Journal of Prosthodontics on Complete and Removable Dentures, 8(1): 18-23.

11. Lam E, Ruprecht A, Yang J (1995): Comparison of two-dimensional orthoradially reformatted computed tomography and panoramic radiography for dental 
implant treatment planning. The Journal of Prosthetic Dentistry, 74(1): 42-46.

12. Al-Ekrish A, Ekram M (2011): A comparative study of the accuracy and reliability of multidetector computed tomography and cone beam computed tomography in the assessment of dental implant site dimensions. Dentomaxillofacial Radiology, 40(2): 67-75.

13. Hashimoto $K$, Kawashima $S$, Araki $M$ et al. (2006): Comparison of image performance between cone-beam computed tomography for dental use and four-row multidetector helical CT. Journal of Oral Science, 48(1): 27-34.

14. Schwarz M, Rothman L, Chafetz N et al. (1987): Computed tomography: Part I. Preoperative assessment of the mandible for endosseous implant surgery. International Journal of Oral \& Maxillofacial Implants, 2(3):137-141.

15. Schwarz M, Rothman S, Rhodes M et al. (1987): Computed tomography: Part II. Preoperative assessment of the maxilla for endosseous implant surgery. International Journal of Oral \& Maxillofacial Implants, 2(3):143-8.

16. Mozzo P, Procacci C, Tacconi A et al. (1998): A new volumetric CT machine for dental imaging based on the cone-beam technique: preliminary results. European Radiology, 8(9): 1558-1564.

17. Arai Y, Tammisalo E, Iwai K et al. (1999): Development of a compact computed tomographic apparatus for dental use. Dentomaxillofacial Radiology, 28(4): 245-248.
18. Araki K, Maki $K$, Seki $K$ et al. (2004): Characteristics of a newly developed dentomaxillofacial X-ray cone beam CT scanner (CB MercuRay $\left.{ }^{\mathrm{TM}}\right)$ : system configuration and physical properties. Dentomaxillofacial Radiology, 33(1): 5159.

19. Kalender W, Fuchs T (2003): On the correlation of pixel noise, spatial resolution and dose in computed tomography: theoretical prediction and verification by simulation and measurement. On the Correlation of Pixel Noise, Spatial Resolution and Dose in Computed Tomography, 2003: 1000-1012.

20. Van Daatselaar A, Van der Stelt $P$, Weenen $J$ (2004): Effect of number of projections on image quality of local CT. Dentomaxillofacial Radiology, 33(6): 361-369

21. Katsumata A, Hirukawa A, Okumura $S$ et al. (2007): Effects of image artifacts on gray-value density in limited-volume cone-beam computerized tomography. Oral Surgery, Oral Medicine, Oral Pathology, Oral Radiology, and Endodontology, 104(6): 829-836.

22. Pawelzik J, Cohnen M, Willers R et al. (2002): A comparison of conventional panoramic radiographs with volumetric computed tomography images in the preoperative assessment of impacted mandibular third molars. Journal of Oral and Maxillofacial Surgery, 60(9): 979-984.

23. Saavedra-Abril J, Balhen-Martín C, Stoopen M et al. (2010): Dental multisection CT for the placement of oral implants: technique and applications. Radiographics, 30(7): 1975-1991 